\title{
V. Note on the ratios which the amounts of substances in radioactive equilibrium bear to one another
}

\section{Hugh Mitchell M.A.}

To cite this article: Hugh Mitchell M.A. (1911) V. Note on the ratios which the amounts of substances in radioactive equilibrium bear to one another , Philosophical Magazine Series 6, 21:121, 40-42, DOI: 10.1080/14786440108637002

To link to this article: http://dx.doi.org/10.1080/14786440108637002

冓 Published online: 21 Apr 2009.

Submit your article to this journal $₫$

Џll Article views: 4

Q View related articles $\sqsubset$ 
Note added Oct. 26, 1910.

Since this paper was written there have appeared two communications by Wulf (Le Radium, June 1910 ; Phys. Zeit. 15 Sept. 191(1). These throw much light on the subject of penetrating radiation and confirn some of the conclusions set forth above.

Wulf, with the vessels he nsed, finds for the penetrating radiation in Holland $g=10$, in Paris $q=6$, and at a height of $300 \mathrm{~m}$. on the Eiffel Tower $q=3.5$.

The reduction in $q$ over a lake was about 4.9 , and a similar reduction was determined at a depth of $12 \mathrm{~m}$. beneath the surface of the water. His interesting experiments seem to establish the terrestrial origin of the penetrating radiation and the diminution of intensity with altitude.

The figures appear to require reduction for values in free a.ir, perhaps on the basis of lead 101), zine 60, aluminiam 52, free air 42.

It will be seen that Wulf's value for $q$, even at Paris, is larger than that calculated in this paper, and that a loss of 40 per cent. for an altitude of $300 \mathrm{~m}$. is less than the loss of 64 per cent. which I calculate for an altitude of $100 \mathrm{~m}$. It will no doubt take time to reconcile these points.

At present it would seem that the penetrating radiation both passes through the air more readily, and also produces more ions than laboratory experiments, with $\mathrm{Ra} C$ and metal screens, lead us to infer. It will, however, be remembered that no direct measurements of the coefficient of absorption of the $\gamma$ rays by gases have yet been made.

A. S. E.

V. Note on the Ratios which the Amounts of Substances in Radioactive Equilitivium bear to one another. By Huan Mirohili, M.A.*

(1) $7 \mathrm{THE}$ law of radioactive change is that the rate of 1 disintegration of an active substance is proportional to the amount of it present. A series of radioactive substances is said to be in equilibrium when the ratio of the amount of one substance to that of any other substance in the series remains unchanged as time goes on. It is known that assuming the disintegration rate of the parent substance to be small in comparison with that of any other in the series, the ratio of the amounts of any two substances in the series is approximately equal to the ratio of their average lives. The present note is intended to show that without

* Communicated by F. Soddy, M.A., F.R.S. 
Sulstances in Radioactive Equilibrium bear to one another. 41 this assumption the theoretically correct deduction from the above law still remains very simple.

(2) Let $\xi_{n}$ be the number of atoms initially and $x_{n}$ the number at time $t$ of a radioactive substance $S_{n}$ wbich changes into a radioactive substance $S_{(n+1)}$ at the rate of $\lambda_{n} x_{n}$ atoms per unit time, where $n=1,2,3 \ldots n$. Then the average life of an atom of $S_{n}$ is $\frac{1}{\lambda_{n}}$ units of time and $x_{n}$ satisfies the differential equation

$$
\frac{d x_{n}}{d t}=\lambda_{n-1} x_{n-1}-\lambda_{n} x_{n},
$$

in which when $n=1, \lambda_{0} x_{0}$ is to be taken as zero.

(3) The general solution of this equation giving the quantity $x_{n}$ of the $n$th substance after time $t$ when the parent substance is initially free from products is

$x_{n}=\xi_{1} \lambda_{1} \lambda_{2} \ldots \lambda_{(n-1)}\left\{\frac{\epsilon^{-\lambda_{1} t}}{\Pi\left(\lambda_{i}-\lambda_{1}\right)}+\frac{e^{-\lambda_{2} t}}{\Pi\left(\lambda_{i}-\lambda_{2}\right)}+\ldots \frac{\epsilon^{-\lambda_{n} t}}{\Pi\left(\lambda_{i}-\lambda_{n}\right)}\right\}$

where the expression $\operatorname{II}\left(\lambda_{i}-\lambda_{x}\right)$ is to be taken to mean the product of all terms of the type $\left(\lambda_{i}-\lambda_{x}\right)$ when $\lambda_{i}$ takes all possible values from $\lambda_{1}$ to $\lambda_{n}$ except $\lambda_{x}$.

For example,

and

$$
x_{1}=\xi_{1} \epsilon^{-\lambda_{1} t}
$$

$$
\begin{aligned}
r_{4} & =\xi_{1} \lambda_{1} \lambda_{2} \lambda_{3}\left\{\frac{\epsilon^{-\lambda_{1} t}}{\left(\lambda_{2}-\lambda_{1}\right)\left(\lambda_{3}-\lambda_{1}\right)\left(\lambda_{4}-\lambda_{1}\right)}+\frac{e^{-\lambda_{2} t}}{\left(\lambda_{1}-\lambda_{2}\right)\left(\lambda_{3}-\lambda_{2}\right)\left(\lambda_{4}-\lambda_{2}\right)}\right. \\
& \left.+\frac{\epsilon^{-\lambda_{3} t}}{\left(\lambda_{1}-\lambda_{3}\right)\left(\lambda_{2}-\lambda_{3}\right)\left(\lambda_{4}-\lambda_{3}\right)}+\frac{\epsilon^{-\lambda_{4} t}}{\left(\lambda_{1}-\lambda_{4}\right)\left(\lambda_{2}-\lambda_{4}\right)\left(\lambda_{3}-\lambda_{4}\right)}\right\} .
\end{aligned}
$$

(4) When the parent substance is the longest lived member of the series, as $t$ increases, $\frac{x_{n}}{x_{1}}$ approaches the value

Now

$$
\frac{\lambda_{1} \lambda_{2} \ldots \lambda_{n-1}}{\left(\lambda_{2}-\lambda_{1}\right)\left(\lambda_{2}-\lambda_{1}\right) \ldots\left(\lambda_{n}-\lambda_{1}\right)}
$$

$$
\begin{gathered}
1+\frac{\lambda_{1}}{\lambda_{8}-\lambda_{1}}+\frac{\lambda_{1} \lambda_{2}}{\left(\lambda_{2}-\lambda_{1}\right)\left(\lambda_{3}-\lambda_{1}\right)}+\ldots \\
+\frac{\lambda_{1} \lambda_{2} \ldots \lambda_{(n-1)}}{\left(\lambda_{2}-\lambda_{1}\right)\left(\lambda_{3}-\lambda_{1}\right) \ldots\left(\lambda_{n}-\lambda_{1}\right)}=\frac{\lambda_{2} \lambda_{3} \ldots \lambda_{n}}{\left(\lambda_{2}-\ddot{\lambda}_{1}\right)\left(\lambda_{3}-\lambda_{1}\right) \ldots\left(\lambda_{n}-\lambda_{1}\right)^{\circ}}
\end{gathered}
$$


Hence

That is

$$
\frac{x_{1}}{x_{1}}+\frac{x_{2}}{x_{1}}+\frac{x_{3}}{x_{1}}+\ldots \frac{x_{n}}{x_{1}}=\frac{\lambda_{n}}{\lambda_{1}} \cdot \frac{x_{n}}{x_{1}}
$$

$$
\frac{x_{n}}{x_{1}+x_{2}+x_{3}+\ldots x_{n}}=\frac{\lambda_{1}}{\lambda_{n}} \text {. }
$$

$\mathrm{H}$ nce in a series of substances in radioactive equilibrium the ratio of the number of atoms of the $n$th substance to those of it and all the preceding substances in coexistence with it is equal to the ratio of the average life of the $n$th substance to that of the first substance present in the series.

High School, Dundee.

VI. A Note on a Method of Determining Capacities in Measurements of Ionization. By Norsan Campbell*.

THE ordinary method of determining the capacity of the 1 ionization vessels and the measuring instrument used in observations on ionization consists in comparing the rate of rise of potential of the electrode system for a constant current (1) when a known capacity is put in parallel with the electrode, and (2) when that capacity is absent. The advantages of the method lie in the simplicity of the apparatus required; its disadvantages are connected with the choice of the standard capacity. Standard condensers of not less than 01 m.t. are easily procured, but their capacity is very great compared with that of the usual measuring systems, which is usually less than 0005 m.f. If they are used, either the time for which the rise of potential is observed in (1) must be so long that difficulties are likely to arise from defective insulation, or the time for which the rise is observed in (2) must be so short that it cannot be measured with accuracy. On the other hand, accurate and well-insulated standard condensers, the capacity of which is of the same order as that of the measuring instrument, are not easily procured, and the (unknown) capacity of the conductors used to connect them to the measuring system may be too large a fraction of their capacity to be safely neglected.

The following method, which appears not to be known generally, is free from these disadvantages. Though there is nothing new in its principle, which is sufficiently obvious, it may be worth while to draw attention to it.

* Communicated by the Author. 